\title{
CONSUMPTIVE HYPOTHYROIDISM: AN UNUSUAL PARANEOPLASTIC MANIFESTATION OF A GASTRIC GASTROINTESTINAL STROMAL TUMOR
}

\author{
T. Patial ${ }^{1, *}$, K. Sharma ${ }^{2}$, D. Thakur ${ }^{3}$, G. Gupta ${ }^{4}$ \\ ${ }^{1}$ Department of General Surgery, Indira Gandhi Medical College, Shimla, Himachal Pradesh 171001, \\ India \\ ${ }^{2}$ Department of Radiation Oncology, Rajiv Gandhi Cancer Institute \& Research Centre, Sector 5, Rohini, \\ New Delhi 110085, Delhi, India \\ ${ }^{3}$ Department of General Surgery, Indira Gandhi Medical College, Shimla, Himachal Pradesh 171001, \\ India \\ ${ }^{4}$ Department of Pathology, School of Medical Sciences \& Research, Sharda University, Greater Noida, \\ Uttar Pradesh 201306, India
}

\begin{abstract}
A 42-year-old hypothyroid shepherd presented with a progressive abdominal lump accompanied by nausea and abdominal fullness. In addition, he had worsening hypothyroidism, despite supranormal doses of thyroxine. Computed tomography of the abdomen was suggestive of a mass lesion in relation to the stomach. A resection of the mass was done and the histopathology was suggestive of gastrointestinal stromal tumor. After surgery, the patient became euthyroid. We believe the patient had consumptive hypothyroidism due to the tumor. Key Words: gastrointestinal stromal tumors, CD117, hypothyroidism, imatinib mesylate, paraneoplastic syndrome.
\end{abstract}

Gastrointestinal stromal tumors (GIST) are mesenchymal tumors that are believed to originate from the interstitial cells of Cajal or from mesenchymal stem cells present throughout the body. Owing to the physical effects of the tumor, the usual presentation can be abdominal discomfort, bleeding, nausea, vomiting, or early satiety. Very rarely do these tumors present with paraneoplastic syndrome. We report the case of a patient who had hypothyroidism due to a GIST of the stomach, which resolved after surgery.

A 42-year-old shepherd was referred to our hospital by his internist with the chief complaints of awareness of a progressive upper abdominal lump for 2 months and postprandial nausea with abdominal fullness for 2 weeks. The patient was a known case of hypothyroidism, and had been on $25 \mu \mathrm{g}$ of thyroxine (T4) for the past 15 months, but for the past 1 month, his serum thyroid stimulating hormone (TSH) levels failed to return to previous levels despite taking $200 \mu \mathrm{g}$ of T4. His most recent thyroid profile at the time was, TSH $49 \mu \mathrm{lU} / \mathrm{ml}$, triiodothyronine (T3) $-0.37 \mathrm{ng} / \mathrm{ml}, \mathrm{T} 4-$ $4.3 \mu \mathrm{g} / \mathrm{dl}$ and reverse T3 $-292.4 \mathrm{ng} / \mathrm{dl}$ (normal range: TSH $-0.30-0.50 \mu \mathrm{lU} / \mathrm{ml}, \mathrm{T} 3-0.60-1.81 \mathrm{ng} / \mathrm{ml}, \mathrm{T} 4-$ 5.01 to $12.45 \mu \mathrm{g} / \mathrm{dl}$ and reverse $\mathrm{T} 3-10-40 \mathrm{ng} / \mathrm{dl}$ ). The patient had been compliant with medication and follow up. The patient was a teetotaller, and had no history of tobacco use. On examination of the abdomen, there was a $10 \times 6 \mathrm{~cm}$ intra-abdominal lump present in the epigastrium, extending to the left hypochondrium, umbilical and the left lumbar regions. The lump was firm, non-tender, non-pulsatile, and mobile. Labora-

Submitted: October 04, 2016.

${ }^{\star}$ Correspondence: E-mail: drtusharpatial@gmail.com Abbreviations used: GIST - gastrointestinal stromal tumors; T3 triiodothyronine; T4 - thyroxine; TSH - thyroid stimulating hormone. tory tests, including a complete blood cell count, blood biochemistry, liver enzymes, and serum amylase and lipase levels, were within normal limits. On computed tomography of the abdomen, a solid mass of size $14.8 \times 9.6 \mathrm{~cm}$ with heterogeneous non-enhancing low attenuation areas was identified in relation to the stomach (Fig. 1). On upper gastrointestinal endoscopy, a submucosal mass lesion was found in relation to the posterior wall of the stomach, without evidence of mucosal involvement. The patient underwent a distal gastrectomy with retrieval of a gastric mass arising from the posterior wall of the stomach without rupture of the mass during handling (Fig. 2). Other solid organs were normal. No lymphadenopathy or ascites was present.

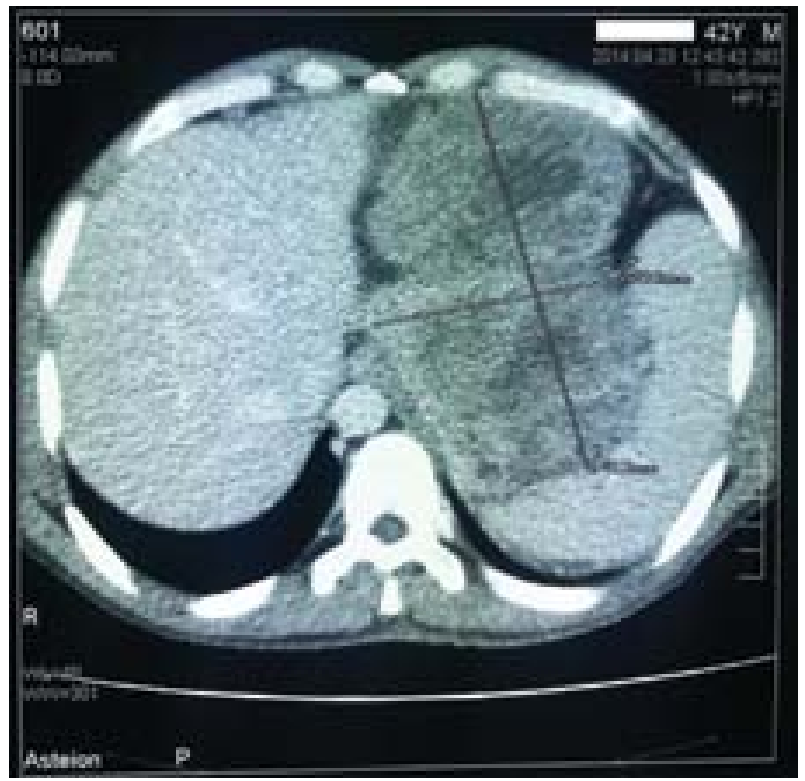

Fig. 1. Computed tomography of the abdomen showing a solid mass of size $14.8 \times 9.6 \mathrm{~cm}$ with heterogeneous non enhancing low attenuation areas identified in relation to the stomach 


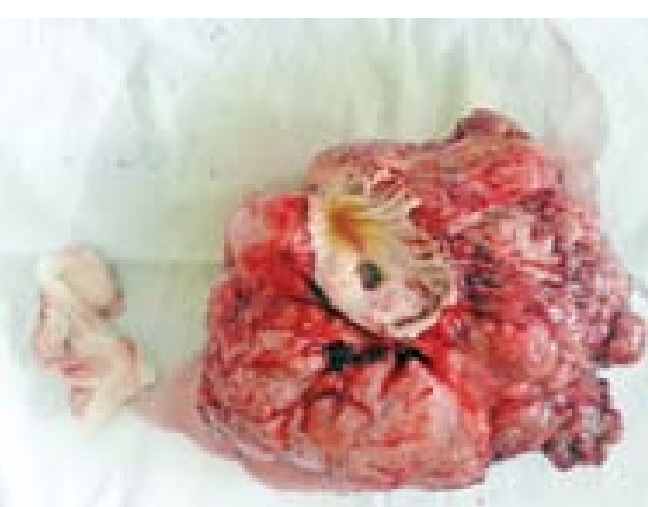

Fig. 2. Gross pathology specimen of the removed GIST, following distal gastrectomy, with tumor free margins

On macroscopic examination, there was a solitary, round tumor measuring $11 \times 8 \times 6 \mathrm{~cm}$ in size. The lesion was well demarcated and had a pseudocapsule. On cut sectioning, the surface was mostly solid with a few cystic areas. Interspersed within were foc of necrosis and hemorrhage. Microscopic evaluation revealed a cellular proliferation of spindle-shaped cells with high nucleus:cytoplasm ratio. Cellular and nuclea pleomorphism was also present. Few areas of necrosis were also seen Mitotic figures were seen occasionally emedded within are of increased mitosis ( $9 / 50$ hig power field) (Fig 3-5). On imun

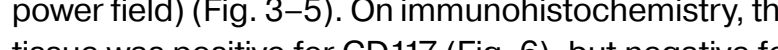
tissue was postive for CDit7 (Fig. 6), but negative for desminat $>10 \mathrm{~cm}$ ), histopathog (mitosis $>5 / 50$ ing (size $>10 \mathrm{~cm}$ ), histopathology (milosis $>5 / 50$ high power field) and immunohistochemistry a diagnosis of the GIST of the stomach with a high risk of neoplastic progression was made. The patient had an uneventful recovery and was discharged 3 days after surgery on adjuvant imatinib mesylate. Post-operative thyroid function tests after 6 weeks were, $\mathrm{TSH}-3.9 \mu \mathrm{IU} / \mathrm{m}$ $\mathrm{T} 3-0.74 \mathrm{ng} / \mathrm{ml}$, T4 $-7.2 \mu \mathrm{g} / \mathrm{dl}$, and reverse T3 $252.3 \mathrm{ng} / \mathrm{dl}$. After gradual reduction of exogenous T4, the drug has been withdrawn and the patient has been euthyroid since.
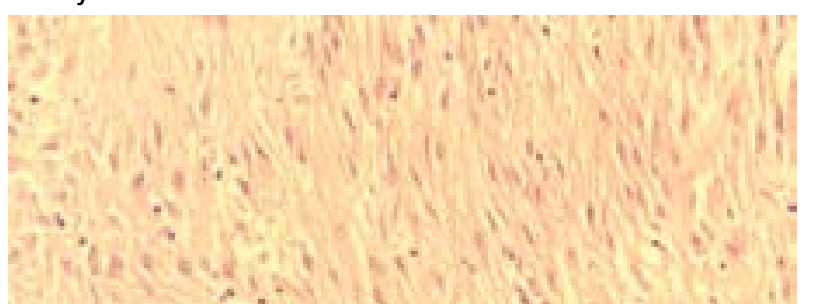

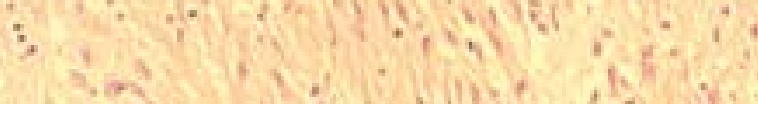
Fig. 3. Microphotograph of gastrectomy specimen (hemato-
xylin-eosin) showing features of a spindle cell type GIST with xylin-eosin) showing fe
nuclear pleomorphism

\section{DISCUSSION}

The median age of GIST presentation is $55-60$ years and these tumors represent $80 \%$ of all mesenchyma

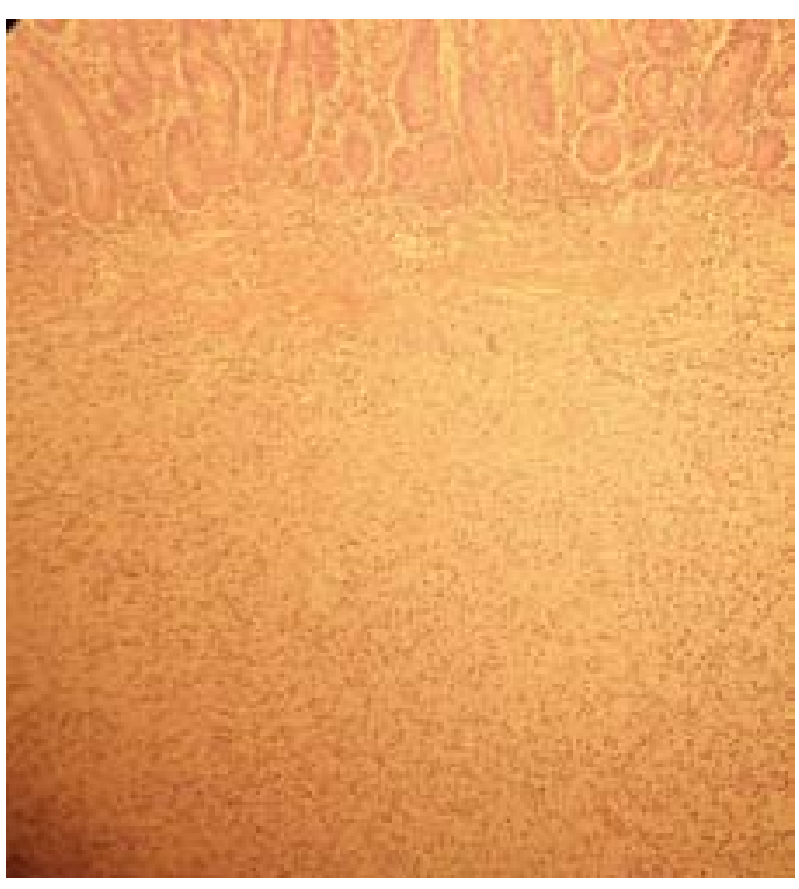

Fig. 4. Microphotograph of specimen (hematoxylin-eosin) showing normal gastric mucosa with underlying spindleshaped cells

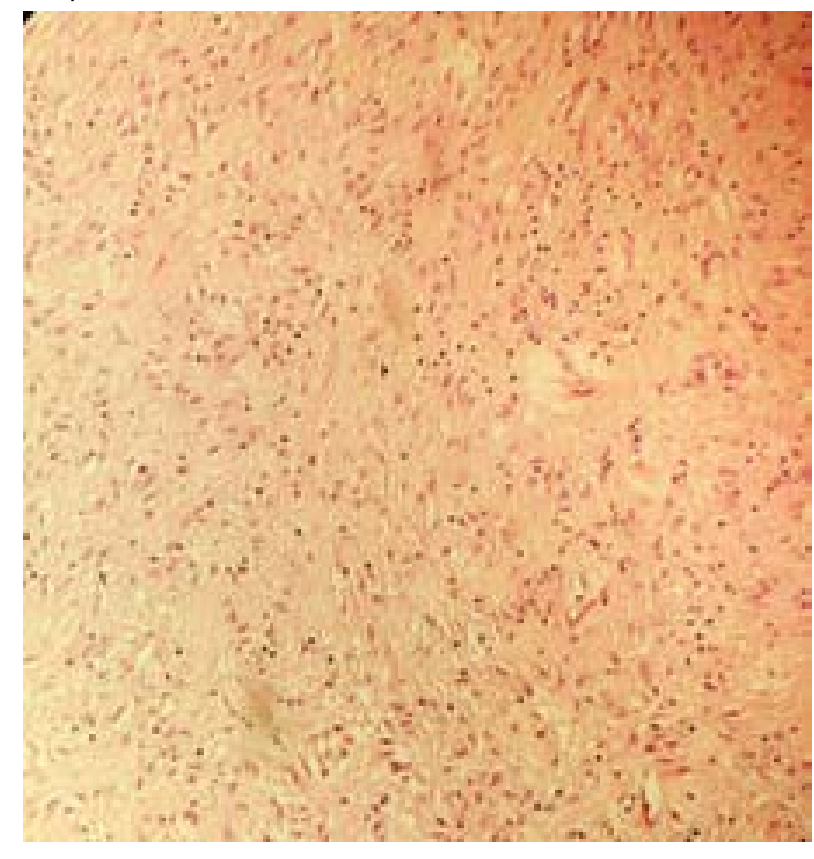
Fig. 5. Microphotograph of th
magnification of microscope

gastrointestinal tumors and $0.1-3 \%$ of all gastrointestinal malignancies [1]. About $70 \%$ of the patients with in $10 \%$ the symptomatic and in $10 \%$ the lesions are detected only at autopsy. The tumor may present as abdominal discomfort, bleeding, nausea, vomiting, or early satiety. Tumor spread is usually intra-abdominal, and metastasis to lymph nodes is rare. Symptoms are usually dependant on the site involved, with the most common being the stomach $(60 \%)$ and the small intestine (30-20\%) [2].

Although rare, paraneoplastic syndromes have been described with GIST. These include autoimmune haemolytic anaemia, hypoglycaemia, hyponatremia nephrotic syndrome, headache and diplopia [3-7]

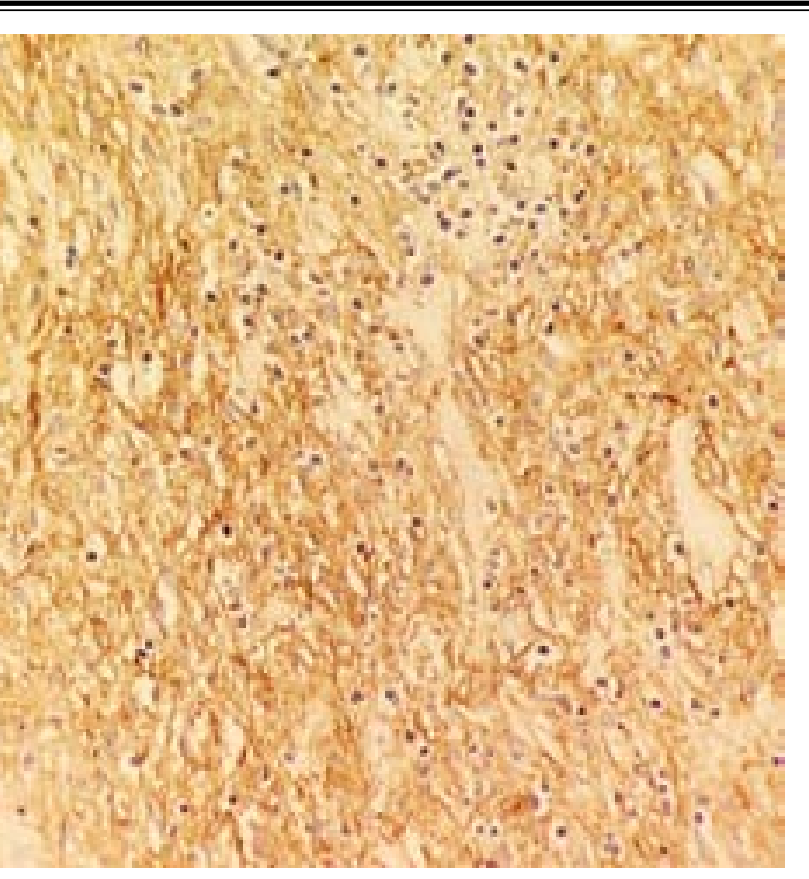

Fig. 6. Microphotograph of CD117 expression in tumor cells

A peculiar consequence of GIST is consumptive hypothyroidism. First reported in children with infantile haemangiomas, this condition results from accelerated degradation of circulating thyroid hormone at rates higher than the synthetic capacity of the normally stimulated thyroid gland. In cases of tyrosine kinase inhibitor naive patients, consumptive hypothyroidism is caused by overexpression of the thyroid hormonenactivating enzyme iodothyronine deiodinase 3 . The diagnosis of consumptive hypothyroidism requires evidence of either elevated levels of serum reverse T3 or supranormal requirements for exogenous thyroid hormone [8]. In our patient, the patient's requirement or supranormal doses of T4 was abolished soon after surgery, thus supporting this diagnosis.

On contrast-enhanced computed tomography, peripheral enhancement is present in the $75 \%$ of the cases of GIST. This represents an enhancement of peripheral areas of viable tumor. However, in a minority of patient's areas of low attenuation may be present corresponding to haemorrhage, necrosis, or cyst formation, as present in our case. We do not believe this is of consequence with respect to consumptive hypothyroidism, as the imaging finding has been described without the paraneoplastic syndrome as well [9].

Surgery has been treatment of choice with $60 \%$ of patients being cured by surgery alone. Laparo- scopic resection is considered a feasible procedure for patients with gastric GIST $5 \mathrm{~cm}$ or smaller, if not contraindicated by comorbidities. Locally advanced GIST may be candidates for surgical resection after neo-adjuvant treatment with Imatinib, a c-Kit inhibitor In cases of inoperable, metastatic or recurrent GIST Imatinib remains the first-line treatment $[1,10]$

Although hypothyroidism is a frequent clinical scenario, the astute clinician must remember that hypothyroidism can often be the herald of a much more sinister diagnosis such as a GIST. In cases of patients requiring supranormal doses of $\mathrm{T} 4$, a measurement of reverse T3 should be considered to establish the diagnosis of consumptive hypothyroidism.

\section{ACKNOWLEDGEMENTS}

Nothing to disclose.

\section{CONFLICT OF INTEREST}

No conflict of interest.

\section{REFERENCES}

1. Caterino S, Lorenzon L, Petrucciani N, et al. Gastroin作 (vitur

2.

2. Rammohan A, Sathyanesan J, Rajendran K, et al. A gist intest Oncos 2013;5:102-12.

3. Alcol 201, 5. 102-12. 3. Alsal 2013; 5 : Mo2-12. I, Nasser H. Autoimmune hemolytic anemia: a rare presentation of gastrointestinal stroma

4. J (1)

5. Bonato V. Lalle M, De Mattia G. Paraneoplastic severe Ayponatremia in a patient with GIST: case report. Endocrine Abstracts 2012; 29: P451.

6. Guirgis HM, Holcombe RF. A case of advanced gastroin6.

7. 7. Ta. Jne K, Midorikawa Y, Yamazaki S, et al. Gastroin. 8. Maynard MA, Marino-Enriquez A, Fletcher JA, et al. Thyroid hormone inactivation in gastrointestinal stromal tumors. N Engl J Med 2014; 370: 1327-34.

9. Afifi AH, Eid M. Gastrointestinal stromal tumors GISTs): diagnostic value of multi-detector computed tomog.

10. Nishida T, Blay JY, Hirota S, et al. The standard dinnosis, treatment, and follow-up of gastrointestinal strom tumors based on guidelines. Gastric Cancer 2016; 19: 3-14. 